\title{
Carotid Arterial Calcium Scoring Using Upper Airway Computed Tomography in Patients with Obstructive Sleep Apnea: Efficacy as a Clinical Predictor of Cerebrocardiovascular Disease
}

Jae Hoon Lee, MD, PhD ${ }^{1 *}$, Eun-Ju Kang, MD, PhD ${ }^{2 *}$, Woo Yong Bae, MD, PhD ${ }^{1}$, Jong Kuk Kim, MD, PhD ${ }^{3}$, Jae Hyung Choi, MD, PhD ${ }^{4}$, Chul Hoon Kim, MD, PhD ${ }^{5}$, Sang Joon Kim, MD, Kyoo Sang Jo, MD ${ }^{1}$, Moon Sung Kim, $M D^{2}$, Tae Kyung Koh, MD, $\mathrm{PhD}^{6}$

Departments of ${ }^{1}$ Otorhinolaryngology-Head and Neck Surgery, ${ }^{2}$ Radiology, ${ }^{3}$ Neurology, ${ }^{4}$ Neurosurgery, and ${ }^{5}$ Oral and Maxillofacial Surgery, Dong-A University College of Medicine, Busan, Korea; ${ }^{6}$ Department of Otorhinolaryngology-Head and Neck Surgery, St. Mary's Medical Center, Busan, Korea

Objective: To evaluate the value of airway computed tomography (CT) in patients with obstructive sleep apnea (OSA) as a predictor of cerebrocardiovascular disease (CCVD) clinically, by quantitatively analyzing carotid arterial calcification (CarAC). Materials and Methods: This study included 287 patients aged 40-80 years, who had undergone both polysomnography (PSG) and airway CT between March 2011 and October 2015. The carotid arterial calcium score (CarACS) was quantified using the modified Agatston method on each upper airway CT. The OSA severity was categorized as normal, mild, moderate, and severe using the PSG results. Clinical characteristics, comorbid diseases, and lipid profiles of all patients were analyzed, and the prevalence of CCVDs was investigated during the follow up period (52.2 \pm 16.0 months).

Results: CCVD occurred in 27 patients $(9.3 \%)$ at the end of follow-up, and the CCVD-present groups showed a significantly older mean age ( 57.5 years vs. 54.2 years), higher prevalence of hypertension (59\% vs. $34 \%$ ) and CarAC (51.9\% vs. 20.8\%), whereas sex, other comorbid diseases, and severity of OSA were not significantly different from the CCVD-absent group. A univariate analysis showed that age, hypertension, incidence of CarAC, and CarACS were risk factors for the occurrence of CCVD events. In a multivariate analysis, the incidence of CarAC was the only independent risk factor for CCVD.

Conclusion: CarAC is an independent risk factor for CCVD, whereas the severity of OSA is not a contributory risk factor in patients with OSA. Therefore, additional analysis of CarACS based on airway CT scans may be useful for predicting CCVD. Keywords: Airway; Calcification; Carotid artery; Computed tomography; Obstructive sleep apnea

\section{INTRODUCTION}

Obstructive sleep apnea (OSA) is a common sleeprelated breathing disorder characterized by recurrent partial or complete obstruction of the upper airway leading to intermittent hypoxia and frequent arousals during sleep.
Many studies have demonstrated that patients with OSA show a relatively higher prevalence of atherosclerotic cerebrovascular and cardiovascular diseases (1-5). A dentist or otolaryngologist uses a cephalometric radiograph to analyze the patient's craniofacial characteristics (retrognathic mandible, narrow palate, tonsillar hypertrophy,

Received August 12, 2018; accepted after revision December 16, 2018.

This study was supported by research funds from Dong-A University.

*These authors contributed equally to this work.

Corresponding author: Woo Yong Bae, MD, PhD, Department of Otorhinolaryngology-Head and Neck Surgery, Dong-A University College of Medicine, 26 Daesingongwon-ro, Seo-gu, Busan 49201, Korea.

- Tel: (8251) 240-2831•Fax: (8251) 253-0712•E-mail: doncamel@dau.ac.kr

This is an Open Access article distributed under the terms of the Creative Commons Attribution Non-Commercial License (https:// creativecommons.org/licenses/by-nc/4.0) which permits unrestricted non-commercial use, distribution, and reproduction in any medium, provided the original work is properly cited. 
and macroglossia) to fabricate an oral appliance for OSA. In some cases, carotid arterial calcification (CarAC) may be identified $(6,7)$. Tsuda et al. (7) reported that subjects showing calcification in the carotid artery area on a cephalometric radiograph have higher Framingham risk scores than those without calcification. They concluded that the presence of CarAC in patients with OSA indicates a cardiovascular risk. However, cephalometry provides a two-dimensional (2D) image; hence, it may be limited to only detecting calcification, as the quantitative analysis of calcification is unavailable. Notably, these authors did not assess the direct relationship between CarAC and the development of a real cardiovascular event. The Agatston's method (Agatston score) has been widely used to assess calcification of coronary arteries and predict cardiovascular events (8-11). Several investigators have reported the feasibility of this method by focusing on quantifying CarAC using a carotid computed tomography (CT) scan (1214). Airway CT is commonly used to evaluate the threedimensional (3D) airway morphology in patients with OSA to detect an anatomically obstructive site (15), and the carotid arteries are always included in the $z$-axis range of this CT scan. Therefore, we performed a quantitative analysis of CarAC using the airway CT scans of OSA patients using the Agatston method. The purpose of this study was to evaluate the clinical value of CarAC as a predictor of cerebrocardiovascular disease (CCVD) in OSA patients.

\section{MATERIALS AND METHODS}

\section{Subjects}

In total, 408 consecutive patients undergoing both polysomnography (PSG) and upper airway CT from March 2011 to October 2015 at our medical center were enrolled. Patients $<40$ years $(n=104)$ or $>80$ years $(n=1)$, and those with an incomplete medical record or lost to followup during the clinical work-up period $(n=2)$ were excluded. Among the 301 subjects included, 14 patients with a history of cardiovascular or cerebrovascular disease (e.g., old myocardial infarction or old stroke) were excluded based on their medical records or direct interviews. Finally, 287 patients (224 males, 63 females; mean age $54.5 \pm 8.2$ years; age range 40-79 years) were enrolled in this study (Fig. 1, Table 1). The institutional ethics committee approved this study and written informed consent was waived.

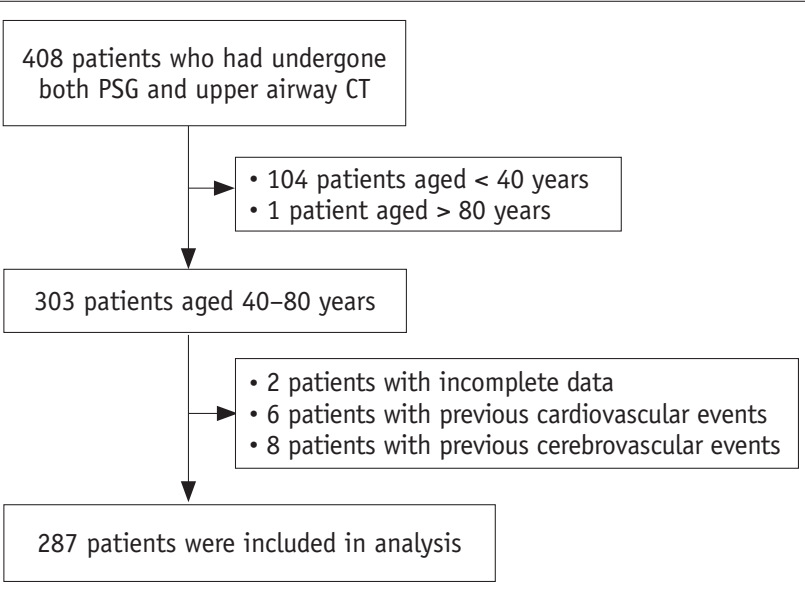

Fig. 1 Patient flow diagram. Total of 408 patients who had undergone PSG and upper airway CT scan and 303 patients aged 40-80 years were included. Sixteen patients were excluded due to incomplete data or history of cardiovascular or cerebrovascular event, and 287 patients were included as final subjects. CT = computed tomography, PSG = polysomnography

\section{Polysomnography}

Patients were diagnosed with OSA based on an overnight PSG (Alice 5, 19 channels; Philips Respironics, Inc., Kennesaw, GA, USA). The PSG is a multi-parametric sleep test, used as a diagnostic tool for OSA, provides simultaneous recordings of multiple physiological parameters related to sleep and wakefulness and directly monitors and quantifies the number of respiratory events, such as obstructive, central, or complex, and the resulting hypoxemia and arousals related to respiratory events.

In this study, apnea was defined as a $>90 \%$ reduction of airflow for at least 10 seconds, and hypopnea as $\geq 30 \%$ decrease in airflow for at least 10 seconds combined with an arousal and/or $\geq 4 \%$ oxygen desaturation. Respiratory effort related arousals (RERAs) were defined as increasing respiratory efforts for at least 10 seconds, followed by an abrupt arousal from deeper sleep, but did not meet the apnea or hypopnea criteria. The apnea-hypopnea index (AHI) is defined as the average number of apnea events plus hypopnea events per hour of sleep. The respiratory disturbance index (RDI) is the average number of combined apnea events, hypopnea events, and RERAs per hour during sleep (RDI = apnea + hypopnea + RERAs per hour of sleep). The RDI was used for severity classification and diagnosis of OSA. The severity of OSA was divided into four groups with respect to the RDI: normal (RDI < 5), mild (RDI 5-14), moderate (RDI 15-29), and severe ( $R D I \geq 30$ ). 


\section{Measurement of Carotid Artery Calcification}

All subjects underwent non-contrast CT scans with a 320-detector-row scanner (Aquilion ONE; Canon Medical Systems Corporation, Otawara, Japan) for an airway evaluation within one week of the PSG examination. The CT scans were performed from the frontal sinus of the skull to

\section{Table 1. Characteristics of Enrolled Subjects}

\begin{tabular}{|c|c|}
\hline No. of patients & 287 \\
\hline Age (years) & $54.5 \pm 8.2$ \\
\hline Male sex (\%) & $224(78.0)$ \\
\hline BMI $\left(\mathrm{kg} / \mathrm{m}^{2}\right)$ & $26.8 \pm 4.7$ \\
\hline \multicolumn{2}{|l|}{ Smoking (\%) } \\
\hline Current & $61(21.0)$ \\
\hline Past & $73(25.5)$ \\
\hline Never & $153(53.5)$ \\
\hline Hypertension* & $104(36.4)$ \\
\hline Systolic BP & $130.2 \pm 16.0$ \\
\hline Diastolic BP & $78.8 \pm 12.9$ \\
\hline Diabetes mellitus ${ }^{\dagger}(\%)$ & $36(12.6)$ \\
\hline Total cholesterol (mg/dL) & $191.5 \pm 44.7$ \\
\hline HDL cholesterol (mg/dL) & $50.5 \pm 12.3$ \\
\hline LDL cholesterol (mg/dL) & $114.4 \pm 36.1$ \\
\hline RDI (event/h) & $30.6 \pm 23.2$ \\
\hline AHI (event/h) & $27.4 \pm 22.7$ \\
\hline RERAs (event/h) & $3.9 \pm 5.1$ \\
\hline ESS & $9.6 \pm 4.6$ \\
\hline Lowest $\mathrm{O}_{2}$ saturation (\%) & $81.5 \pm 9.8$ \\
\hline \multicolumn{2}{|l|}{ OSA groups (\%) } \\
\hline Normal (RDI < 5) & $41(14.3)$ \\
\hline Mild $(5 \leq \mathrm{RDI}<15)$ & $42(14.6)$ \\
\hline Moderate $(15 \leq \mathrm{RDI}<30)$ & $74(25.8)$ \\
\hline Severe $(30 \leq \mathrm{RDI})$ & $130(45.3)$ \\
\hline Carotid artery calcification & $68(23.7)$ \\
\hline CarACS & $32.6 \pm 129.6$ \\
\hline $\log (\operatorname{CarACS}+1)$ & $0.386 \pm 0.774$ \\
\hline \multicolumn{2}{|l|}{ CarACS groups (\%) } \\
\hline $0-10$ & $235(81.9)$ \\
\hline $11-100$ & $34(11.8)$ \\
\hline $101-400$ & $11(3.8)$ \\
\hline$>400$ & $7(2.4)$ \\
\hline
\end{tabular}

Data are expressed as mean \pm standard deviation or numbers of patients (\%). * Patients were considered to have hypertension if their BP was persistently $>140 / 90 \mathrm{~mm} \mathrm{Hg}$ or if they were currently taking antihypertensive medication, ${ }^{\dagger}$ Patients were considered to have diabetes mellitus if their fasting glucose level was $\geq 126 \mathrm{mg} /$ $\mathrm{dL}$, as assessed at least once, or if they were currently taking oral hypoglycemic agents or insulin. AHI = apnea-hypopnea index, BMI $=$ body mass index, $\mathrm{BP}=$ blood pressure, $\mathrm{Car} \mathrm{ACS}=$ carotid arterial calcium score, ESS = Epworth Sleepiness Scale, HDL = high-density lipoprotein, $\mathrm{LDL}=$ low-density lipoprotein, $\mathrm{OSA}=$ obstructive sleep apnea, RDI = respiratory disturbance index, RERAs = respiratory effort related arousals the carina level of the distal trachea, to include both nasal and intra-thoracic airways (Fig. 2A). The airway CT scan included the following parameters: collimation $320 \times 0.5$ $\mathrm{mm}$, gantry rotation time $500 \mathrm{~ms}$, tube voltage $120 \mathrm{kV}$ tube voltage, tube current $80 \mathrm{~mA}$, and slice thickness $0.5 \mathrm{~mm}$. All datasets were processed with iterative reconstruction (AIDR 3D, Canon Medical Systems Corporation) with a 3-mm slice thickness and a 3-mm interval. The images were then transferred to commercial software (Vitrea 6.0; Vital Images, Minnetonka, MN, USA) for post-processing and analysis. The carotid arterial calcium scores (CarACS) were quantified using the modified Agatston method, as reported in previous studies $(11,13)$. On axial CT images, the software automatically highlights densities $>130$ Hounsfield units in an area of $1 \mathrm{~mm}^{3}$ (Fig. 2B). The evaluation of CarAC was performed by an experienced radiologist with particular attention to exclude bony spurs or calcified ligamentous structures, and sum of the calcium score was quantified automatically by the software. CarACS was measured in the common, external, and internal carotid arteries, and total CarACS was estimated as the sum of these scores.

\section{Clinical Analysis}

The clinical histories of the subjects were reviewed retrospectively within one month of the CT study, which included the history of comorbid diseases (e.g., hypertension, diabetes mellitus, and smoking), lipid profile (total cholesterol, high-density lipoprotein [HDL] and lowdensity lipoprotein [LDL]), blood pressure, body weight/ height, and body mass index (BMI). A history of CCVD of each subject was closely evaluated based on the medical record or direct interview for exclusion.

In this study, CCVD events were defined by the development of certain conditions after PSG and upper airway CT examinations, such as stroke, transient ischemic attack (TIA), myocardial infarction, unstable angina with significant coronary stenosis in patients undergoing coronary arterial intervention (coronary stent insertion or coronary arterial bypass graft surgery), and cerebrovascular or cardiovascular death (death caused by ischemic heart disease or stroke and sudden death). Stroke was defined as a focal neurological deficit lasting $>24$ hours with a clinically relevant lesion found on brain imaging without any non-vascular cause identified. TIA was defined as a focal neurological deficit lasting 30 seconds to 24 hours without the brain imaging suggesting stroke. The date of a CCVD event or date and cause of death were obtained by 


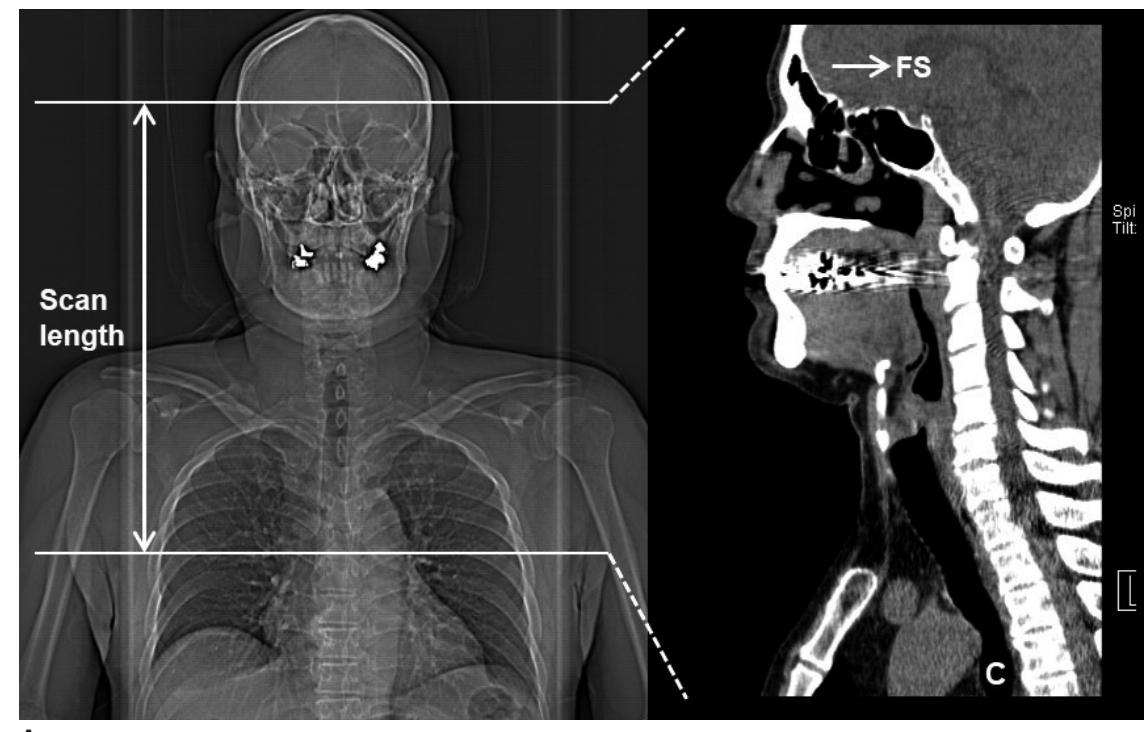

A

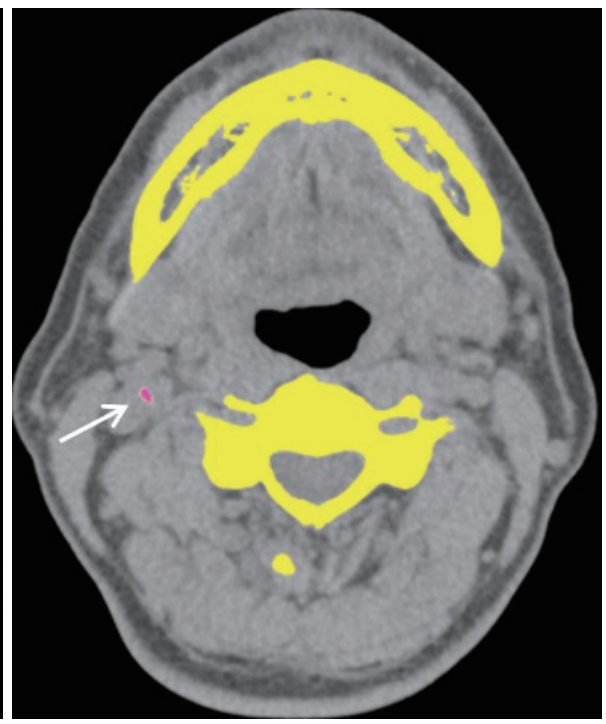

B

Fig. 2 Representative images of airway CT scans.

(A) CT scans starts from frontal sinus of skull ('FS') to carina level of distal trachea ('C'), including both upper airway and trachea; (B) Semiautomatic measurement of CarAC for calculating Agatston score; software automatically highlights calcification (coding to yellow color) with CT number higher than 130 Hounsfield units. Following this, reader determines carotid arterial calcium (arrow) based on anatomical information on each of CT images, and sum of calcium score is automatically quantified using software. CarAC = carotid arterial calcification

reviewing the hospital records. The follow-up period ranged from 31-80 months, and the mean follow-up period was $52.2 \pm 16.0$ (mean \pm standard deviation) months.

\section{Statistics}

The various clinical characteristics were compared among patients with and without CCVD events. The independent $t$ test was used for continuous variables, and Fisher's exact test was used for binomial variables. The Mann-Whitney U-test was performed for non-normally distributed variables, according to the results produced by the Kolmogorov-Smirnov test. The CarACS was analyzed using logarithmic transformation after adding a constant of 1 to each score due to the wide range of values. Risk factors for outcomes were first examined using a univariate Cox proportional hazards model, and variables with a significant association $(p<0.20)$ were applied to a multivariate Cox proportional hazards model. Cumulative incidence curves for CCVD events were estimated by the Kaplan-Meier method and evaluated by log-rank test. Statistical Package for Social Sciences (SPSS version 20.0; IBM Corp., Armonk, NY, USA) was used for all data analyses, and $p$ values $<0.05$ were considered significant.

\section{RESULTS}

Among the selected 287 patients, CCVD events occurred in $27(9.3 \%)$ at the end of the follow-up period. One patient developed myocardial infarction and cerebral stroke simultaneously, 13 patients had a cardiovascular event, and the remaining 13 developed cerebrovascular events. The details of subjects with CCVD events have been summarized in Table 2.

The CCVD event-present (+) group showed a significantly higher mean age than the CCVD event-absent (-) group $(57.5 \pm 7.7$ vs. $54.2 \pm 8.2, p=0.047)$. The total number of patients with hypertension was 104 among the 287 subjects $(36.4 \%)$, and the prevalence of hypertension was significantly higher in the CCVD event (+) group than the CCVD event (-) group (59.3\% [16/27] vs. $34.0 \%$ [88/260], $p=0.017)$. Sex, lipid profiles (total cholesterol, HDL, and LDL), BMI, smoking history, and RDI were not different between the CCVD events (+) and CCVD events (-) groups (Table 3).

The PSG results of the subjects are summarized in Table 4. The mean AHI, RERA, Epworth Sleepiness Scale, and lowest $\mathrm{O}_{2}$ saturation were not different between the two groups. OSA severity was divided into four groups (normal, mild, moderate, and severe) using RDI, and the numbers of subjects in each group were, normal OSA $=39$; mild OSA = 42; moderate $\mathrm{OSA}=74$; and severe $\mathrm{OSA}=132$.

Mild, moderate, and severe OSA groups showed similar percentages of CCVD events occurrence (5/42 [11.9\%], $8 / 74$ [10.8\%], and 14/132 [10.6\%], respectively), while 
Table 2. Subjects with CCVD Events

\begin{tabular}{|c|c|c|c|}
\hline Subjects & CCVD Events & CarACS & RDI \\
\hline$M / 73$ & UA (1-vessel, stent) & 471.86 & 57.4 \\
\hline$M / 70$ & Stroke (Lt. MCA) & 283.59 & 32.9 \\
\hline$M / 68$ & UA (2-vessel, stent) & 37.46 & 19.6 \\
\hline$M / 66$ & Stroke (Rt. PICA) & 75.01 & 33.5 \\
\hline$F / 66$ & Stroke (medullar) & 0.00 & 18.3 \\
\hline$M / 65$ & UA (2-vessel, stent) & 39.29 & 46.0 \\
\hline$F / 63$ & Stroke (Rt. MCA) & 0.73 & 28.6 \\
\hline$F / 63$ & Stroke (Rt. MCA) & 3.12 & 24.5 \\
\hline$M / 62$ & MI (1-vessel, stent), TIA & 594.86 & 18.8 \\
\hline$F / 62$ & Stroke (Lt. basal ganglia) & 0.00 & 55.8 \\
\hline$M / 59$ & TIA (Rt. lacunar) & 0.00 & 21.4 \\
\hline$M / 59$ & UA (1-vessel, stent) & 7.20 & 37.8 \\
\hline$M / 58$ & MI (1-vessel, CABG) & 135.79 & 12.7 \\
\hline$M / 57$ & UA (1-vessel, stent) & 12.20 & 13.2 \\
\hline$M / 55$ & UA (1-vessel, stent) & 18.01 & 52.2 \\
\hline$F / 55$ & TIA (vertebral artery) & 0.00 & 36.9 \\
\hline$F / 54$ & UA (1-vessel, stent) & 0.00 & 48.7 \\
\hline$M / 53$ & UA (1-vessel, stent) & 0.00 & 38.3 \\
\hline$M / 53$ & Stroke (cerebellum) & 0.00 & 5.7 \\
\hline$M / 52$ & Stroke (Lt. MCA) & 0.00 & 31.1 \\
\hline$M / 52$ & UA (2-vessel, stent) & 28.35 & 11.3 \\
\hline$M / 50$ & Stroke (medullar) & 0.00 & 21.5 \\
\hline$M / 50$ & Stroke (medullar) & 0.00 & 29.1 \\
\hline$M / 49$ & UA (1-vessel, stent) & 0.00 & 84.2 \\
\hline$M / 49$ & UA (3-vessel, CABG) & 49.5 & 35.0 \\
\hline$M / 46$ & Stroke (medullar) & 0.00 & 71.9 \\
\hline$M / 43$ & UA (1-vessel, stent) & 0.00 & 7.0 \\
\hline
\end{tabular}

$\mathrm{CABG}=$ coronary arterial bypass graft, $\mathrm{CCVD}=$ cerebrocardiovascular disease, $F=$ female, $L t .=$ left, $M=$ male, $M C A=$ middle cerebral artery, MI = myocardial infarction, PICA = posterior inferior cerebellar artery, Rt. = right, TIA = transient ischemic attack, UA = unstable angina the normal group reported no such events (0/39). However, the proportions of each number of patients according to severity of OSA were not significantly different between the CCVD event (+) group and CCVD event (-) group. CarAC was found in 68 patients $(23.7 \%, 68 / 287)$, mean CarACS was $32.6 \pm 129.6$, and the mean $\log$ transformed calcium score $[“ \log (\operatorname{CarACS}+1)$ "] was $0.386 \pm 0.774$. The CCVD event $(+)$ group showed significantly larger $\log (\mathrm{Car} A C S+1)$ than the CCVD event (-) group $(0.819 \pm 0.979$ vs. $0.341 \pm 0.737, p=$ 0.002 ) (Table 5). The incidence of CarAC was 51.9\% (14/27) in patients with CCVD events, which was significantly higher than in those with no CCVD events $(20.8 \%, 54 / 260, p<0.001)$.

A univariate analysis using the Cox hazards model showed that age (odds ratio [OR], 1.047; $p=0.050$ ), hypertension $(0 R, 2.826 ; p=0.012)$, presence of CarAC (OR, 4.108; $p<0.001)$, and log transformed CarACS (OR, 1.824; $p=$ 0.004 ) were risk factors for CCVD events (Table 6). In the multivariate analysis, the presence of CarAC was the only independent risk factor for CCVD events (OR, 3.692; 95\% confidence interval, 1.382-9.865: $p=0.009)$. The result of Kaplan-Meier analysis comparing the cumulative incidence curve according to the presence or absence of CarAC showed a significant difference (Log-rank test, $p=0.001$ ) (Fig. 3).

\section{DISCUSSION}

OSA is a common disorder that is potentially harmful to health with immediate effects including intermittent hypoxia, fragmented sleep, and substantial influences

Table 3. Baseline Characteristics of Subjects with and without CCVD Events

\begin{tabular}{|c|c|c|c|}
\hline Variables & CCVD Event Absence & CCVD Event Presence & $P$ \\
\hline No. of patient & 260 & 27 & \\
\hline Age (years) & $54.2 \pm 8.2$ & $57.5 \pm 7.7$ & 0.047 \\
\hline Male sex $(\%)$ & $203(78.1)$ & $21(77.8)$ & 1.000 \\
\hline BMI $\left(\mathrm{kg} / \mathrm{m}^{2}\right)$ & $26.8 \pm 4.8$ & $26.8 \pm 3.3$ & 0.979 \\
\hline Smoking (\%) & & & 0.290 \\
\hline Current & $54(20.8)$ & $6(22.2)$ & \\
\hline Past & $63(24.3)$ & $10(37.0)$ & \\
\hline Never & $142(54.8)$ & $11(40.7)$ & \\
\hline Hypertension* & $88(34.0)$ & $16(59.3)$ & 0.017 \\
\hline Systolic BP & $130.5 \pm 16.1$ & $126.7 \pm 15.6$ & 0.246 \\
\hline Diastolic BP & $79.3 \pm 12.9$ & $74.4 \pm 12.1$ & 0.068 \\
\hline 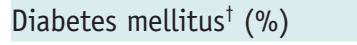 & $31(12.0)$ & $5(18.5)$ & 0.507 \\
\hline Total cholesterol (mg/dL) & $193.1 \pm 44.9$ & $180.4 \pm 33.4$ & 0.238 \\
\hline HDL cholesterol (mg/dL) & $50.8 \pm 12.2$ & $48.9 \pm 13.3$ & 0.574 \\
\hline LDL cholesterol (mg/dL) & $115.7 \pm 37.0$ & $108.5 \pm 32.2$ & 0.459 \\
\hline
\end{tabular}

Data are expressed as mean \pm standard deviation or numbers of patients (\%). *Patients were considered to have hypertension if their BP was persistently $>140 / 90 \mathrm{~mm} \mathrm{Hg}$ or if they were currently taking antihypertensive medication, ${ }^{\dagger}$ Patients were considered to have diabetes mellitus if their fasting glucose level was $\geq 126 \mathrm{mg} / \mathrm{dL}$, as assessed at least once, or if they were currently taking oral hypoglycemic agents or insulin. 
Table 4. Polysomnographic Results of Subjects with and without CCVD Events

\begin{tabular}{lccc}
\hline \multicolumn{1}{c}{ Variables } & CCVD Event Absence & CCVD Event Presence & $P$ \\
\hline No. of patient & 260 & 27 & \\
RDI (event/h) & $34.3 \pm 22.5$ & $33.1 \pm 19.4$ & 0.566 \\
AHI (event/h) & $30.7 \pm 22.4$ & $29.4 \pm 19.2$ & 0.591 \\
RERAs (event/h) & $3.9 \pm 5.1$ & $3.4 \pm 4.9$ & 0.750 \\
ESS & $9.6 \pm 4.6$ & $9.6 \pm 4.7$ & 0.927 \\
Lowest $0_{2}$ saturation (\%) & $81.3 \pm 10.0$ & $83.1 \pm 8.5$ & 0.322 \\
OSA groups (\%) & $39(15.0)$ & & 0.191 \\
$\quad$ Normal $(\mathrm{RDI}<5)$ & $37(14.2)$ & $5(18.5)$ & \\
Mild $(5 \leq \mathrm{RDI}<15)$ & $66(25.4)$ & $8(29.6)$ & \\
Moderate $(15 \leq \mathrm{RDI}<30)$ & $118(45.4)$ & $14(51.8)$ & \\
Severe $(30 \leq \mathrm{RDI})$ & & \\
\hline
\end{tabular}

Data are expressed as mean \pm standard deviation or numbers of patients (\%).

Table 5. CarACS of Subjects with and without CCVD Events

\begin{tabular}{lccr}
\hline \multicolumn{1}{c}{ Variables } & $\begin{array}{c}\text { CCVD Event } \\
\text { Absence }\end{array}$ & $\begin{array}{c}\text { CCVD Event } \\
\text { Presence }\end{array}$ & $P$ \\
\hline No. of patient & 260 & 27 & \\
CarAC (\%) & $54(20.8)$ & $14(51.9)$ & $<0.001$ \\
CarACS & $29.2 \pm 127.3$ & $65.0 \pm 148.5$ & 0.173 \\
Log(CarACS+1) & $0.341 \pm 0.737$ & $0.819 \pm 0.979$ & 0.002 \\
CarACS groups (\%) & & & 0.013 \\
0-10 & $219(84.2)$ & $16(59.3)$ & \\
$11-100$ & $27(10.4)$ & $7(25.9)$ & \\
$101-400$ & $9(3.5)$ & $2(7.4)$ & \\
$>400$ & $5(1.9)$ & $2(7.4)$ & \\
\hline
\end{tabular}

Data are expressed as mean \pm standard deviation or numbers of patients (\%). CarAC: carotid arterial calcification

on blood pressure and intrathoracic pressure, leading to hypertension, cardiovascular events, stroke, depression, and impaired quality of life $(1-5,16-19)$. The OSA prevalence rate has increased substantially according to an American study reported in 2013, and the current prevalence estimates of moderate to severe OSA (AHI $\geq 15)$ are $10 \%$, $17 \%, 3 \%$, and $9 \%$ in 30-49-year-old males, $50-70$-yearold males, 30-49-year-old females, and 50-70-year-old females, respectively (20). Although there is some debate about the effect of OSA treatment on the development of cardiovascular or cerebral events, many patients with OSA visit sleep apnea clinics for management and obtaining counseling for nasal continuous positive airway pressure (CPAP), which is the first-line OSA therapy (21-23). Therefore, physicians (dentists or otolaryngologists) in a sleep clinic may be concerned not only about managing the airway, but also about the management or consultation planning of comorbid diseases. Recently, airway CT has often been used to evaluate the 3D airway morphology in OSA patients before planning the treatment or during the follow-up period (15, 24-26). The use of lateral cephalometric radiographs is limited, as it only provides $2 \mathrm{D}$ images of the airway. However, CT scans provide a detailed analysis of the relationship between the upper airway and its surrounding soft tissues (such as pharyngeal size at various levels of nasopharynx, oropharynx, and hypopharynx in patients with different dentofacial skeletal pattern), and dimensional analysis of the soft palate and tongue to determine the linear, volumetric, and cross-sectional area measurements for evaluation of the interaction of upper airway size and dentofacial structures. In the same manner, airway CT is routinely used in sleep clinics simultaneously with PSG in OSA patients. Therefore, we could analyze the CarACS retrospectively without additional radiation exposure.

In our study, the incidence of CCVD events in OSA patients was $9.3 \%$ at the end of follow-up, with similar incidences of cardiovascular and cerebrovascular events (4.9\%). These incidence rates were consistent or slightly lower than prior studies: Gottlieb et al. (1) reported a 10.6\% (473/4422) incidence of coronary heart disease, and Munoz et al. (4) reported $5.1 \%$ (20/394) incidence of ischemic stroke. We speculated that the causes of this incidence difference of CCVD were the relatively short follow-up period of our study and the data from a single medical center, which might have underestimated the CCVD events. Although the CCVD events were not evaluated in non-OSA subjects in this study, a recent retrospective population-based follow up study reported that one cohort had a 1.95fold higher incidence of major adverse cardiac events compared to a non-OSA cohort (27). Various mechanisms have been reported regarding the relationship between OSA and vascular disease $(20,28-30)$. One review article 
Table 6. Risk Factors for CCVD Events by Cox Analysis

\begin{tabular}{|c|c|c|c|c|c|c|}
\hline \multirow{2}{*}{ Variable } & \multicolumn{3}{|c|}{ Univariate } & \multicolumn{3}{|c|}{ Multivariate* } \\
\hline & OR & $95 \% \mathrm{CI}$ & $P$ & OR & $95 \%$ CI & $P$ \\
\hline Age (years) & 1.047 & $1.001-1.097$ & 0.050 & 1.014 & $0.958-1.074$ & 0.630 \\
\hline Male & 1.018 & $0.392-2.641$ & 0.971 & & & \\
\hline BMI $\left(\mathrm{kg} / \mathrm{m}^{2}\right)$ & 0.999 & $0.916-1.090$ & 0.984 & & & \\
\hline Smoking & 2.049 & $0.828-5.072$ & 0.121 & & & \\
\hline Hypertension & 2.826 & $1.258-6.351$ & 0.012 & 2.021 & $0.840-4.860$ & 0.116 \\
\hline Diabetes mellitus & 1.664 & $0.588-4.713$ & 0.338 & & & \\
\hline Total cholesterol & 0.993 & $0.943-1.002$ & 0.235 & & & \\
\hline RDI (event/h) & 0.995 & $0.977-1.013$ & 0.565 & & & \\
\hline CarAC & 4.108 & $1.824-9.255$ & $<0.001$ & 3.692 & $1.382-9.865$ & 0.009 \\
\hline $\log ($ CarACS+1) & 1.824 & $1.215-2.737$ & 0.004 & 1.494 & $0.910-2.455$ & 0.113 \\
\hline
\end{tabular}

*Multivariate model includes variables for which $p<0.20$ by univariate analysis. CI: confidence interval, OR: odds ratio

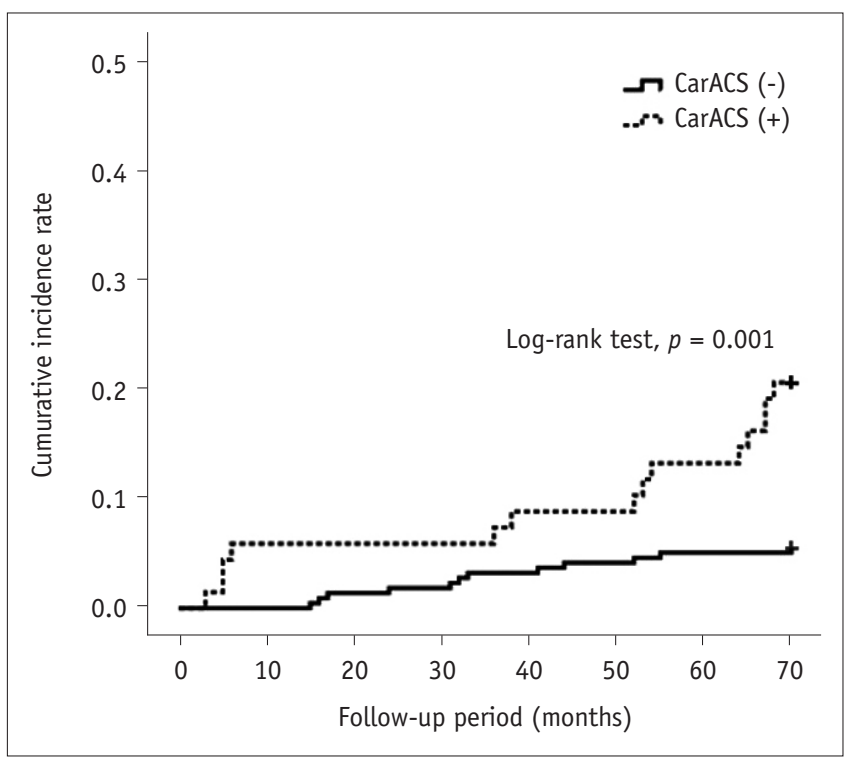

Fig. 3. Kaplan-Meier analysis of cerebrocardiovascular disease events of 287 patients according to presence or absence of CarAC (CarACS). Comparison between incidence curve proved to be significant (Log-rank test, $p=0.001)$. CarACS $=$ carotid arterial calcium score

suggested three possible biological mechanisms supporting the association between OSA, endothelial dysfunction, and arterial disease: intermittent hypoxia leading to increased oxidative stress, systemic inflammation, and sympathetic activity; changes in intrathoracic pressure leading to excessive mechanical stress on the heart and large artery walls; and arousal-induced reflex sympathetic activation with consequent repetitive rises in blood pressure (30).

Majority of the previous studies have used panoramic dental radiographs to evaluate CarAC and its prevalence in the general dental population was 0.8-9\% (31-33). Tsuda et al. (6) evaluated the CarAC prevalence in cephalometric radiographs among OSA patients and detected a higher prevalence of CarAC than the general population (9.5\% vs. $6.7 \%$ ). To the best of our knowledge, this is the first study that investigates CarAC in OSA patients using CT, and the prevalence of CarAC was relatively higher than that reported by prior studies (23.7\%). This was probably because of the use of CT with higher detectability of calcification than the radiographic images, and the difference in the inclusion criteria for the study population (subjects $\geq 40$ ).

Several investigators have reported that the presence of CarAC is an important marker of vascular risk. Cohen et al. (34) reported that $34 \%$ of patients with CarAC on a panoramic radiograph suffered cardiovascular or cerebrovascular events. Moreover, Friedlander et al. (35) evaluated the prevalence of CarAC on panoramic radiographs of recent stroke patients, and found its presence in $37 \%$ of subjects. In a population-based study, a positive correlation was detected between CarAC and CarACS estimated by CT images (36). The CarACS is an accepted method to evaluate coronary heart disease risk and is a subclinical atherosclerosis marker for the prediction of future cardiovascular events (9-11). The prognostic value of CarACS for incident stroke remains controversial; however, according to recently published meta-analysis study, despite the low the incidence of stroke ( $0.26 \%$ /year), the presence of coronary calcification predicts approximately three times higher likelihood for incident stroke as compared to absence of calcifications (37-40). Based on this, we speculated that CarAC might also be associated with cardiovascular events, besides cerebral accidents.

In our study, the prevalence of CarAC (CarACS > 0) was $51.9 \%(14 / 27)$ in patients with CCVD events, which was significantly higher than in patients without CCVD events. However, only $40.7 \%(11 / 27)$ of patients had CarACS > 10 . 
We speculated that the main cause of relatively low CarACS in the majority of patients with CCVD events could be the relatively young age of patients (among 27 patients, 17 were $<60$ years). Some studies reported that in comparison to their age-matched population, younger people with OSA had a higher risk of CCVD than the older $(41,42)$. On the other hand, several studies reported that the non-calcified plaque showed higher vulnerability to develop cerebral or myocardial infarction than calcified plaques. Moreover, some investigators suggested that calcified portions of plaques are in a relatively stable state and seldom rupture (43). Our study adopted a non-contrast CT scan protocol for the evaluation of the upper airway; therefore, we could not evaluate the luminal status or plaque characteristics of the carotid arteries.

We found that the CCVD events were associated with CarAC/CarACS in addition to conventional risk factors (age and hypertension) in OSA patients. However, OSA severity did not contribute significantly to CCVD events. OSA has been considered as a risk factor for CCVD events; however, it remains unclear whether OSA is an independent or accelerating factor (coexisting with other atherogenic risk factors) for increased CCVD risk (1-5). In our study, the presence of OSA was associated with a higher risk of CCVD, but the severity of OSA did not significantly contribute to CCVD events. The direct relationship between the severity of OSA and the development of CCVD is debatable; however, the association of severe untreated OSA with diminished probability of long-term survival has been confirmed in large-cohort population-based studies $(44,45)$. Since the number of subjects in each severity group was uneven and relatively small in our study, we could not control the baseline characteristics of each group. Thus, we speculated that the conventional atherogenic risk factors, such as age or hypertension frequently coexisting in the population with OSA, could have more influence on CCVD events than the OSA severity. Furthermore, the disease duration or treatment status of OSA and the medication for other comorbid diseases may contribute to the potential longterm risk of CCVD.

The association between CarAC and CCVD events persisted even after the traditional atherogenic risk parameters were adjusted, whereas the effect of age, hypertension, and CarACS disappeared. Arterial calcification occurs during the atherosclerotic process, thus, CarAC may potentially reflect all associated parameters for CCVD development. Therefore, in our study, CarAC was an independent risk factor for CCVD events in OSA patients. These results will be important for managing comorbid risk factors for atherosclerosis to prevent CCVD in OSA patients, and to treat OSA in sleep clinics effectively.

There were several limitations of in our study. First, the number of subjects was relatively small and the proportion of patients in each OSA severity category was uneven, which might have affected the statistical results. Second, females were underrepresented in this study with lower prevalence of OSA. It has long been recognized that males have greater vulnerability than females towards developing OSA. Clinic-based studies have shown that in patients referred for clinical evaluation, the ratio of male to female ranged from 5 to 8:1 $(46,47)$. We consecutively included all the OSA patients who had visited our sleep clinic without any exclusion for the female sex, and the sex proportion of our subjects was consistent with previously reported studies. Third, the follow-up period for CCVD events was relatively short and differed among the subjects. Lastly, CPAP and other treatments for OSA or comorbid diseases were not taken into consideration.

In conclusion, the CarAC was an independent risk factor in the occurrence of CCVD events in OSA patients, whereas the RDI did not contribute as a risk factor. This study suggests that additional analyses of CarACS on airway CT in OSA patients may provide information for successful prediction of CCVD.

\section{Conflicts of Interest}

The authors have no potential conflicts of interest to disclose.

\section{ORCID iDs}

Woo Yong Bae

https://orcid.org/0000-0001-5578-0225

Jae Hoon Lee

https://orcid.org/0000-0001-8452-5901

Eun-Ju Kang

https://orcid.org/0000-0003-0937-3607

\section{REFERENCES}

1. Gottlieb DJ, Yenokyan G, Newman AB, O'Connor GT, Punjabi NM, Quan SF, et al. Prospective study of obstructive sleep apnea and incident coronary heart disease and heart failure: the sleep heart health study. Circulation 2010;122:352-360

2. Beaudin AE, Waltz X, Hanly PJ, Poulin MJ. Impact of 
obstructive sleep apnea and intermittent hypoxia on cardiovascular and cerebrovascular regulation. Exp Physiol 2017;102:743-763

3. Maeder MT, Schoch OD, Rickli H. A clinical approach to obstructive sleep apnea as a risk factor for cardiovascular disease. Vasc Health Risk Manag 2016;12:85-103

4. Munoz R, Duran-Cantolla J, Martínez-Vila E, Gallego J, Rubio $R$, Aizpuru F, et al. Severe sleep apnea and risk of ischemic stroke in the elderly. Stroke 2006;37:2317-2321

5. Elkholy SH, Amer HA, Nada MM, Nada MA, Labib A. Sleeprelated breathing disorders in cerebrovascular stroke and transient ischemic attacks: a comparative study. J Clin Neurophysiol 2012;29:194-198

6. Tsuda H, Almeida FR, Tsuda T, Moritsuchi Y, Lowe AA. Cephalometric calcified carotid artery atheromas in patients with obstructive sleep apnea. Sleep Breath 2010;14:365-370

7. Tsuda H, Moritsuchi Y, Almeida FR, Lowe AA, Tsuda T. The relationship between cephalometric carotid artery calcification and Framingham risk score profile in patients with obstructive sleep apnea. Sleep Breath 2013;17:10031008

8. Agatston AS, Janowitz WR, Hildner FJ, Zusmer NR, Viamonte M Jr, Detrano R. Quantification of coronary artery calcium using ultrafast computed tomography. J Am Coll Cardiol 1990;15:827-832

9. Mahabadi AA, Möhlenkamp S, Lehmann N, Kälsch H, Dykun I, Pundt N, et al.; Heinz Nixdorf recall study investigators. CAC score improves coronary and CV risk assessment above statin indication by ESC and AHA/ACC primary prevention guidelines. JACC Cardiovasc Imaging 2017;10:143-153

10. Genders TS, Pugliese F, Mollet NR, Meijboom WB, Weustink AC, van Mieghem CA, et al. Incremental value of the CT coronary calcium score for the prediction of coronary artery disease. Eur Radiol 2010;20:2331-2340

11. Elias-Smale SE, Proença RV, Koller MT, Kavousi M, van Rooij FJ, Hunink MG, et al. Coronary calcium score improves classification of coronary heart disease risk in the elderly: the Rotterdam study. J Am Coll Cardiol 2010;56:1407-1414

12. Denzel C, Lell M, Maak M, Höckl M, Balzer K, Müller KM, et al. Carotid artery calcium: accuracy of a calcium score by computed tomography-an in vitro study with comparison to sonography and histology. Eur J Vasc Endovasc Surg 2004;28:214-220

13. Miralles M, Merino J, Busto M, Perich X, Barranco C, VidalBarraquer F. Quantification and characterization of carotid calcium with multi-detector CT-angiography. Eur J Vasc Endovasc Surg 2006;32:561-567

14. Katano H, Yamada K. Analysis of calcium in carotid plaques with Agatston scores for appropriate selection of surgical intervention. Stroke 2007;38:3040-3044

15. Kaur S, Rai S, Kaur M. Comparison of reliability of lateral cephalogram and computed tomography for assessment of airway space. Niger J Clin Pract 2014;17:729-736

16. Somers VK, White DP, Amin R, Abraham WT, Costa F, Culebras
A, et al. Sleep apnea and cardiovascular disease: an American Heart Association/American College of Cardiology Foundation scientific statement from the American Heart Association council for high blood pressure research professional education committee, council on clinical cardiology, stroke council, and council on cardiovascular nursing. J Am Coll Cardiol 2008;52:686-717

17. Yaffe K, Laffan AM, Harrison SL, Redline S, Spira AP, Ensrud $K E$, et al. Sleep-disordered breathing, hypoxia, and risk of mild cognitive impairment and dementia in older women. JAMA 2011;306:613-619

18. Silva GE, An MW, Goodwin JL, Shahar E, Redline S, Resnick H, et al. Longitudinal evaluation of sleep-disordered breathing and sleep symptoms with change in quality of life: the Sleep Heart Health Study (SHHS). Sleep 2009;32:1049-1057

19. Peppard PE, Szklo-Coxe M, Hla KM, Young T. Longitudinal association of sleep-related breathing disorder and depression. Arch Intern Med 2006;166:1709-1715

20. Peppard PE, Young T, Barnet JH, Palta M, Hagen EW, Hla KM. Increased prevalence of sleep-disordered breathing in adults. Am J Epidemiol 2013;177:1006-1014

21. Wang X, Zhang Y, Dong Z, Fan J, Nie S, Wei Y. Effect of continuous positive airway pressure on long-term cardiovascular outcomes in patients with coronary artery disease and obstructive sleep apnea: a systematic review and meta-analysis. Respir Res 2018;19:61

22. Anandam A, Patil M, Akinnusi M, Jaoude P, El-Solh AA. Cardiovascular mortality in obstructive sleep apnoea treated with continuous positive airway pressure or oral appliance: an observational study. Respirology 2013;18:1184-1190

23. Peker Y, Strollo PJ Jr. CPAP did not reduce cardiovascular events in patients with coronary or cerebrovascular disease and moderate to severe obstructive sleep apnoea. Evid Based Med 2017;22:67-68

24. Chousangsuntorn K, Bhongmakapat T, Apirakkittikul N, Sungkarat W, Supakul N, Laothamatas J. Computed tomography characterization and comparison with polysomnography for obstructive sleep apnea evaluation. J Oral Maxillofac Surg 2018;76:854-872

25. Rodrigues MM, Pereira Filho VA, Gabrielli MFR, Oliveira TFM, Batatinha JAP, Passeri LA. Volumetric evaluation of pharyngeal segments in obstructive sleep apnea patients. Braz J Otorhinolaryngol 2018;84:89-94

26. Fleck RJ, Ishman SL, Shott SR, Gutmark EJ, McConnell KB, Mahmoud M, et al. Dynamic volume computed tomography imaging of the upper airway in obstructive sleep apnea. J Clin Sleep Med 2017;13:189-196

27. Lin YS, Liu PH, Chu PH. Obstructive sleep apnea independently increases the incidence of heart failure and major adverse cardiac events: a retrospective population-based follow-up study. Acta Cardiol Sin 2017;33:656-663

28. Lui MM, Sau-Man M. OSA and atherosclerosis. J Thorac Dis 2012;4:164-172

29. Curry BD, Bain JL, Yan JG, Zhang LL, Yamaguchi M, Matloub 
HS, et al. Vibration injury damages arterial endothelial cells. Muscle Nerve 2002;25:527-534

30. Kohler M, Stradling JR. Mechanisms of vascular damage in obstructive sleep apnea. Nat Rev Cardiol 2010;7:677-685

31. Lewis DA, Brooks SL. Cartoid artery calcification in a general dental population: a retrospective study of panoramic radiographs. Gen Dent 1999;47:98-103

32. Kumagai M, Yamagishi T, Fukui N, Chiba M. Carotid artery calcification seen on panoramic dental radiographs in the asian population in Japan. Dentomaxillofac Radiol 2007;36:92-96

33. Brand HS, Mekenkamp WC, Baart JA. Prevalence of carotid artery calcification on panoramic radiographs. Ned Tijdschr Tandheelkd 2009;116:69-73

34. Cohen SN, Friedlander AH, Jolly DA, Date L. Carotid calcification on panoramic radiographs: an important marker for vascular risk. Oral Surg Oral Med Oral Pathol Oral Radiol Endod 2002;94:510-514

35. Friedlander AH, Manesh F, Wasterlain CG. Prevalence of detectable carotid artery calcifications on panoramic radiographs of recent stroke victims. Oral Surg Oral Med Oral Pathol 1994;77:669-673

36. Odink $A E$, van der Lugt $A$, Hofman $A$, Hunink MG, Breteler MM, Krestin GP, et al. Association between calcification in the coronary arteries, aortic arch and carotid arteries: the Rotterdam study. Atherosclerosis 2007;193:408-413

37. Wong ND, Gransar H, Shaw L, Polk D, Moon JH, MirandaPeats $\mathrm{R}$, et al. Thoracic aortic calcium versus coronary artery calcium for the prediction of coronary heart disease and cardiovascular disease events. JACC Cardiovasc Imaging 2009;2:319-326

38. Gibson A0, Blaha MJ, Arnan MK, Sacco RL, Szklo M,
Herrington DM, et al. Coronary artery calcium and incident cerebrovascular events in an asymptomatic cohort. The MESA Study. JACC Cardiovasc Imaging 2014;7:1108-1115

39. Hermann DM, Gronewold J, Lehmann N, Moebus S, Jöckel $\mathrm{KH}$, Bauer $\mathrm{M}$, et al. Coronary artery calcification is an independent stroke predictor in the general population. Stroke 2013;44:1008-1013

40. Chaikriangkrai K, Jhun HY, Palamaner Subash Shantha G, Bin Abdulhak A, Sigurdsson G, Nabi F, et al. Coronary artery calcium score as a predictor for incident stroke: systematic review and meta-analysis. Int J Cardiol 2017;236:473-477

41. Peker Y, Hedner J, Norum J, Kraiczi H, Carlson J. Increased incidence of cardiovascular disease in middle-aged men with obstructive sleep apnea: a 7-year follow-up. Am J Respir Crit Care Med 2002;166:159-165

42. Lavie P, Lavie L, Herer P. All-cause mortality in males with sleep apnoea syndrome: declining mortality rates with age. Eur Respir J 2005;25:514-520

43. Thomas IC, Forbang NI, Criqui MH. The evolving view of coronary artery calcium and cardiovascular disease risk. Clin Cardiol 2018;41:144-150

44. Young T, Finn L, Peppard PE, Szklo-Coxe M, Austin D, Nieto FJ, et al. Sleep disordered breathing and mortality: eighteen-year follow-up of the Wisconsin sleep cohort. Sleep 2008;31:10711078

45. Punjabi NM, Caffo BS, Goodwin JL, Gottlieb DJ, Newman AB, $0^{\prime}$ Connor GT, et al. Sleep-disordered breathing and mortality: a prospective cohort study. PLoS Med 2009;6:e1000132

46. Strohl KP, Redline S. Recognition of obstructive sleep apnea. Am J Respir Crit Care Med 1996;154:279-289

47. Punjabi NM. The epidemiology of adult obstructive sleep apnea. Proc Am Thorac Soc 2008;5:136-143 